\title{
Tunable ohmic environment using Josephson junction chains
}

\author{
Gianluca Rastelli ${ }^{1}$ and Ioan M. Pop ${ }^{2}$ \\ ${ }^{1}$ Zukunftskolleg \& Fachbereich Physik, Universität Konstanz, 78457 Konstanz, Germany \\ ${ }^{2}$ Physikalisches Institut, Karlsruhe Institute of Technology, 76131 Karlsruhe, Germany
}

(Received 20 December 2017; revised manuscript received 13 April 2018; published 17 May 2018)

\begin{abstract}
We propose a scheme to implement a tunable, wide frequency-band dissipative environment using a double chain of Josephson junctions. The two parallel chains consist of identical superconducting quantum interference devices (SQUIDs), with magnetic-flux tunable inductance, coupled to each other at each node via a capacitance much larger than the junction capacitance. Thanks to this capacitive coupling, the system sustains electromagnetic modes with a wide frequency dispersion. The internal quality factor of the modes is maintained as high as possible, and the damping is introduced by a uniform coupling of the modes to a transmission line, itself connected to an amplification and readout circuit. For sufficiently long chains, containing several thousands of junctions, the resulting admittance is a smooth function versus frequency in the microwave domain, and its effective dissipation can be continuously monitored by recording the emitted radiation in the transmission line. We show that by varying in situ the SQUIDs' inductance, the double chain can operate as a tunable ohmic resistor in a frequency band spanning up to $1 \mathrm{GHz}$, with a resistance that can be swept through values comparable to the resistance quantum $R_{q}=h /\left(4 e^{2}\right) \simeq 6.5 \mathrm{k} \Omega$. We argue that the circuit complexity is within reach using current Josephson junction technology.
\end{abstract}

DOI: 10.1103/PhysRevB.97.205429

\section{INTRODUCTION}

Dissipation in radiofrequency (rf) superconducting quantum electronic circuits [1-3] is usually detrimental, giving rise to quantum decoherence. However, this does not necessarily have to be the case. Remarkably, in the past decade, engineered dissipation [4-7] played an increasingly prominent role in quantum states stabilization [8-12] or even in error correction schemes [13-15].

So far, low-impedance dissipative environments have dominated the scene, as they are ubiquitously present in rf circuits and can be tailored using standard microwave design strategies. Designing high impedance environments, with an impedance comparable to the resistance quantum $R_{q}=$ $h /\left(4 e^{2}\right) \simeq 6.5 \mathrm{k} \Omega$, has proven more challenging. Recently, significant success has been achieved in the fabrication of low-loss high impedance environments in the form of superinductors [16-20] or metamaterials $[21,22]$. However, the implementation of wide frequency-band, high-impedance ohmic environments remains an unsolved problem.

Tunable, high-impedance ohmic environments are potentially interesting for several applications in the field of superconducting electronics. For instance, quantum simulations of fundamental models to study dissipative quantum phase transitions require the exploration of extended regions in their phase diagrams [23,24]. In a single Josephson junction, dissipation leads to a phase transition with suppression of the quantum tunneling of the superconducting phase when the effective resistance shunting the junction is swept through the resistance quantum $R_{q}$. The phase diagram of such a transition was experimentally explored using different shunting resistances $[25,26]$. In circuit QED, the ratio between the characteristic impedance $Z_{c}$ of a one-dimensional (1D) microwave waveguide and the quantum resistance $R_{q}$ plays the role of the effective fine-structure constant between the artificial atoms, viz. superconducting qubits, and the electromagnetic field [27], namely $\alpha_{\text {eff }}=\left(Z_{c} / Z_{\mathrm{vac}}\right) \alpha \sim Z_{c} / R_{q}$, with the $Z_{\mathrm{vac}}$ the impedance of the vacuum and $\alpha \simeq 1 / 137$.

An ultrastrong-coupling regime in circuit QED has been achieved in experiments in resonant cavities $[28,29]$ and in open microwave waveguides [30], using galvanic coupling, which is characterized by a dual scaling of the coupling strength in matter-radiation interaction [27,31], e.g., $\sim 1 / \alpha_{\text {eff }}$. This regime was also obtained experimentally in the effective rotating frame of a driven qubit coupled to an $\mathrm{LC}$ resonator [32], with a theoretical extension to an ensemble of resonators [33]. Hence, circuit QED designs offer another realization of the spin-boson model, a reference model in the theory of quantum dissipation. For instance, a recent experiment investigated transmons coupled to transmission lines with different coupling strength [34]. Another recent approach is based on the use of 1D arrays of Josephson junctions to design the resonant modes of the electromagnetic environment $[35,36]$. In these systems, it is desirable to have the ability to controllably sweep the relevant parameter over a wide range, i.e., the strength of the dissipative interaction between the quantum system and its environment [37-39]. Varying in situ the resistance opens the route for addressing novel issues such as, for instance, quenching in the dissipative phase transition by varying rapidly the external dissipation across the critical point.

This class of environments could also be an asset for quantum state preparation and stabilization $[40,41]$ and autonomous quantum error correction via bath engineering [42,43]. For example, in the context of coherent cat state preparation, tuning the dissipative strength and the characteristic impedance might provide a significant resource [42-44]. 


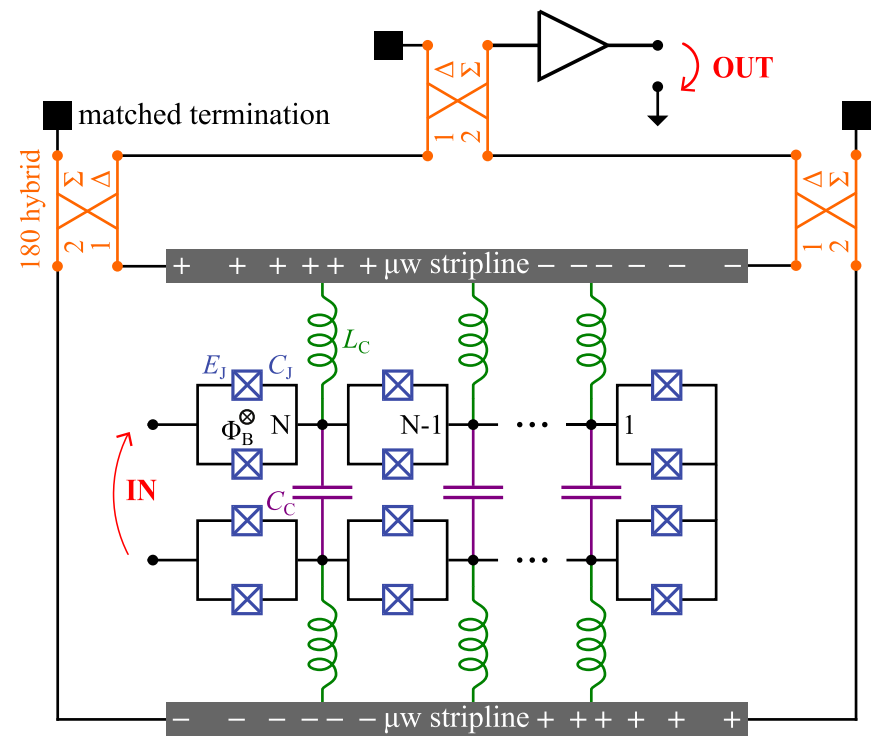

FIG. 1. Schematic of the two parallel Josephson junction chains (PJJCs). The effective Josephson junctions are implemented using SQUIDs threaded by a magnetic flux $\Phi_{B}$ to achieve a tunable Josephson inductance $L_{J}=\Phi_{0}^{2} /\left[8 \pi^{2} E_{J} \cos \left(2 \pi \Phi_{B} / \Phi_{0}\right)\right]$, where $\Phi_{0}=$ $h /(2 e)$ is the flux quantum. The two chains are coupled via the capacitances $C_{C}$, with $C_{C} \gg C_{J}$. Each node is inductively coupled through the inductance $L_{C}$ to a balanced microstrip transmission line. The transmission line is connected via $180^{\circ}$ hybrid couplers to a standard coaxial line, ideally without reflections, and the signal can then be routed to a microwave amplification and readout chain.

In this work, we analyze the possibility of realizing a tunable high-impedance environment, ohmic in a wide frequency range, using two coupled parallel Josephson junction chains (PJJCs), as depicted in Fig. 1. Josephson junctions (JJs) are versatile circuit elements, with widespread use in quantum mesoscopic systems, thanks to their intrinsic low dissipation and amenable nonlinearity. They are the building blocks of superconducting quantum bits (qubits) [45-47], hybrid systems $[48,49]$, or Josephson photonic circuits [50,51]. Josephson junction chains exhibit rich and interesting many-body physical properties [52], which can be influenced relatively accurately by circuit design and fabrication parameters. They have constituted the platform of choice for the investigation of quantum fluctuations of the phase induced by charge interactions, i.e., quantum phase slips [53-63], or quantum fluctuations of the charge induced by Josephson tunneling [64-71].

In the phase regime, where the Josephson energy $E_{J}=$ $\hbar I_{c} /(2 e)$, with $I_{c}$ the junction critical current, dominates over the charging energy of the junction $E_{C}=e^{2} /\left(2 C_{J}\right)$, with $C_{J}$ the junction capacitance, Josephson junction chains have already been investigated as custom-designed electromagnetic environments, implementing metamaterials [72-77], resonators with tunable nonlinearity $[78,79]$, or parametric amplifiers [80]. The success of many-junction devices in the phase regime $\left(E_{J} \gg E_{C}\right)$ paves the way toward more complex architectures, such as the two coupled Josephson junction chains we propose in Fig. 1 to implement a tunable, highimpedance ohmic environment.
The PJJC device shown in Fig. 1 consists of two JJ chains capacitively coupled to each other at each node and inductively coupled to a stripline microwave transmission line. Each element is formed by a superconducting quantum interference device (SQUID), with $E_{J} \gg E_{C}$, threaded by a magnetic flux $\Phi_{B}$ that allows tuning of the Josephson inductance $L_{J}=\Phi_{0}^{2} /\left[8 \pi^{2} E_{J} \cos \left(2 \pi \Phi_{B} / \Phi_{0}\right)\right]$. The coupling capacitance between the chains $C_{C}$ is designed to be dominant compared to $C_{J}\left(C_{J} \ll C_{C}\right)$, which imposes a dense and linear dispersion relation over a wide frequency range. Dissipation is introduced via the inductive coupling (using $L_{C}$ ) of the chains to an on-chip microwave transmission line, which is itself connected to an amplification and read-out circuit using $180^{\circ}$ hybrid couplers to mode-match between the on-chip transmission line and the standard $50 \Omega$ coaxial cable. This matching is important to avoid the formation of standing waves in the transmission line, which would result in a nonuniform coupling of the PJJC eigenmodes to the $50 \Omega$ environment.

We show that for sufficiently long chains, with $N$ in the range of $10^{3}$, the resulting real part of the impedance at the input port of the PJJC is a smooth function versus frequency in a band of $\sim 1 \mathrm{GHz}$, and its value $\simeq \sqrt{L_{J} / C_{C}}$ is tunable in situ, straddling the resistance quantum. Additionally, due to the fact that dissipation is introduced via coupling to a transmission line, one can continuously monitor the photons emitted by the device of interest, connected to the input port of the PJJC.

Notice that, in our proposal, Josephson junctions are only used as linear inductances and could be in principle replaced by geometric inductors. Nevertheless, Josephson inductors are very convenient for this proposal, as they offer three essential ingredients: (a) an intrinsically lossless medium, (b) an ultracompact inductor, much larger than the geometric inductance of an equivalent size wire, and (c) tunability via the Josephson effect, when implemented in the shape of a SQUID.

The paper is structured as follows. In Sec. II we discuss the admittance of a single $\mathrm{JJ}$ chain. We compare a phenomenological model for dissipation, based on an infinite number of dissipationless junctions, with models for finite-size JJ chains, formed by $N$ dissipative junctions. In Sec. III we analyze the effective circuit of the PJJC in Fig. 1 and show its equivalence to a single chain formed by $N$ JJs. In Sec. IV, we discuss the realistically achievable values for the admittance of the PJJC device, taking into account the limited range of experimentally feasible parameters. Finally, we draw our conclusions in Sec. V.

\section{ADMITTANCE OF A SINGLE CHAIN FORMED BY $N$ LUMPED ELEMENTS}

In Sec. II A we recall the emergence of an ohmic resistor in the mathematical limit of an infinite line formed by dissipationless JJs acting as linear inductances and capacitances. In Sec. II B we demonstrate that a similar result can be obtained for finite chain lengths, $N$, if the $\mathrm{JJ}$ element of the chain is intrinsically dissipative. In a first example, assuming typical measured values for the intrinsic dissipation of the $\mathrm{JJ}$, the real part of the resulting admittance can only become a smooth function versus frequency for chain lengths of the order $N=$ $10^{5}$. In a second example, we engineer the dissipation, and we can obtain a smooth admittance versus frequency for much 


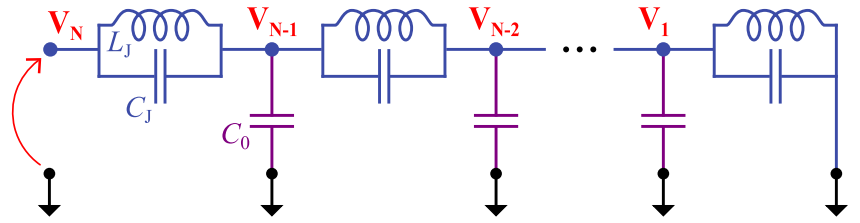

FIG. 2. Model for a single chain formed by an inductance $L_{J}$ in parallel to a capacitances $C_{J}$. Each island is connected by a capacitance $C_{0}$ to the ground.

shorter chains with $N \sim 10^{3}$. The latter results will be directly applicable to the PJJC device (as shown in Sec. III).

\section{A. Ohmic admittance of a dissipationless $\mathbf{J J}$ chain in the thermodynamic limit}

We consider the chain shown in Fig. 2 formed by a series of inductances $L_{J}$, in parallel with capacitances $C_{J}$, with $C_{0}$ connecting each node to the ground. Introducing the two admittances $Y_{J}(\omega)=i \omega C_{J}+1 /\left(i \omega L_{J}\right)$ and $Y_{0}(\omega)=i \omega C_{0}$, we write Kirchhoff's laws for current conservation at nodes $n=1, \ldots, N$, in terms of the voltages $v_{n}=V_{n}(\omega)$, in the frequency domain

$Y_{J}(\omega)\left(v_{n}-v_{n-1}\right)=Y_{J}(\omega)\left(v_{n-1}-v_{n-2}\right)+Y_{0}(\omega) v_{n}$,

with the boundary condition $V_{0}=v_{0}=0$. We consider a vector of dimension $N-1$ composed of the voltage values at nodes $n=1, \ldots, N-1$. Then, Eq. (1) can be cast in the following tridiagonal matrix form:

$$
\left(\begin{array}{ccccc}
a(\omega) & -1 & 0 & \ldots & \ldots \\
-1 & a(\omega) & -1 & 0 & \ldots \\
0 & -1 & a(\omega) & -1 & \ldots \\
\ldots & \ldots & \ldots & \ldots & -1 \\
\ldots & \ldots & 0 & -1 & a(\omega)
\end{array}\right)\left(\begin{array}{c}
v_{N-1} \\
v_{N-2} \\
\ldots \\
\ldots \\
\ldots \\
v_{1}
\end{array}\right)=\left(\begin{array}{c}
v_{N} \\
0 \\
\ldots \\
\ldots \\
\ldots \\
0
\end{array}\right)
$$

with $a(\omega)=2+Y_{0}(\omega) / Y_{J}(\omega)$.

The previous matrix has eigenvalues $\lambda_{k}(\omega)=$ $2[1-\cos (\pi k / N)]+Y_{0}(\omega) / Y_{J}(\omega)$ and eigenvectors $e_{k}(n)=$ $\sqrt{2 / N} \sin (\pi k n / N)$ for $k \in[1, N-1]$ defined on the restricted lattice $n \in[1, N-1]$. The eigenvectors are orthonormal, $\sum_{n=1}^{N-1} e_{k}(n) e_{k^{\prime}}(n)=\delta_{k k^{\prime}}$, and satisfy the completeness relation $\sum_{k=1}^{N-1} e_{k}(n) e_{k}(m)=\delta_{n m}$. The matrix appearing on the left-hand side of Eq. (2) can be written as $\overline{\bar{Y}}=\overline{\bar{U}} \overline{\bar{D}} \overline{\bar{U}}^{-1}$, where $\overline{\bar{D}}$ is the diagonal matrix of the eigenvalues, and $\overline{\bar{U}}$ $\left(\overline{\bar{U}}^{-1}\right)$ is a matrix whose $k$-row (-column) are the components of the eigenvector $k$. By writing the inverse of the matrix as $\overline{\bar{Y}}^{-1}=\overline{\bar{U}} \overline{\bar{D}}^{-1} \overline{\bar{U}}^{-1}$, one can express the voltage at node $N-1$ as a function of the voltage at node $N$, namely $v_{N-1}=v_{N} \sum_{k=1}^{N-1} e_{k}^{2}(n) / \lambda_{k}(\omega)$, which reads

$$
v_{N-1}=v_{N} \frac{2}{N} \sum_{k=1}^{N-1} \frac{Y_{J}(\omega) \sin ^{2}(\pi k / N)}{Y_{0}(\omega)+2 Y_{J}(\omega)[1-\cos (\pi k / N)]} .
$$

The admittance of the chain is defined by the relation

$$
I(\omega)=Y_{J}(\omega)\left(v_{N}-v_{N-1}\right) \equiv Y_{\mathrm{ch}}(\omega) v_{N} .
$$

Inserting Eq. (3) into Eq. (4), using the relation $\sin ^{2}(x)=[1-$ $\cos (x)][1+\cos (x)]$, and the sum $\sum_{k=1}^{N-1}[1+\cos (\pi k / N)]=$ $N-1$, the admittance can be expressed as

$$
Y_{\mathrm{ch}}(\omega)=\frac{Y_{J}(\omega)}{N}\left(1+\sum_{k=1}^{N-1} \frac{Y_{0}(\omega)\left[1+\cos \left(\frac{\pi k}{N}\right)\right]}{Y_{0}(\omega)+2 Y_{J}(\omega)\left[1-\cos \left(\frac{\pi k}{N}\right)\right]}\right) .
$$

We can cast the admittance $Y_{\mathrm{ch}}(\omega)$ of Eq. (5) as the sum of three terms

$$
Y_{\mathrm{ch}}(\omega)=\frac{1}{i \omega N L_{J}}+i \omega \tilde{C}+Y_{\mathrm{har}}(\omega) .
$$

$Y_{\text {ch }}(\omega)$ is characterized by an effective inductance $N L_{J}$ at small frequency and an effective capacitance $\tilde{C}$ at large frequency, given by

$$
\tilde{C}=\frac{C_{J}}{N}+\frac{C_{0}}{2 N} \sum_{k=1}^{N-1} \frac{\cos ^{2}\left(\frac{\pi k}{2 N}\right)}{\sin ^{2}\left(\frac{\pi k}{2 N}\right)+C_{0} /\left(4 C_{J}\right)} .
$$

This same result was obtained using a different method in previous works [59,81], with different boundary conditions [82]. The third term $Y_{\text {har }}(\omega)$ in Eq. (6) is related to the electromagnetic eigenmodes of the chain, whose spectrum reads

$$
\omega_{k}=\frac{2 \omega_{0} \sin [\pi k /(2 N)]}{\sqrt{1+\left(4 C_{J} / C_{0}\right) \sin ^{2}[\pi k /(2 N)]}} .
$$

We introduce the characteristic frequencies of the spectrum

$$
\omega_{0}=\frac{1}{\sqrt{L_{J} C_{0}}}, \quad \omega_{J}=\frac{1}{\sqrt{L_{J} C_{J}}}, \quad \omega_{m}=\max _{k}\left\{\omega_{k}\right\},
$$

corresponding, respectively, to the frequency scale in the linear regime, the plasma frequency of the single $\mathrm{JJ}$, and the maximum frequency of the spectrum, given by $\omega_{m}=$ $2 \omega_{0} / \sqrt{1+4 C_{J} / C_{0}}$ for $N \gg 1$. Using the eigenmodes spectrum, $Y_{\text {har }}(\omega)$ can be written as

$$
Y_{\mathrm{har}}(\omega)=\frac{i 2 \omega}{N L_{J}} \sum_{k=1}^{N-1} \frac{\left(1-\frac{\omega_{k}^{2}}{\omega_{J}^{2}}\right)\left(1-\frac{\omega_{k}^{2}}{\omega_{m}^{2}}\right)}{\omega_{k}^{2}-\omega^{2}-2 i \varepsilon \omega_{k}} .
$$

In Eq. (10) we added phenomenologically an imaginary part $\varepsilon>0$ in the denominator, which yields a finite real part for the admittance. From Eq. (8) for the modes, and from their corresponding admittance in Eq. (10), in the limit of $\varepsilon=0$, we can recover previous theoretical results $[59,81]$.

It is now interesting to discuss the behavior of $Y_{\text {har }}(\omega)$ at small frequency, with $\pi k /(2 N) \ll 1$, such that we can assume a linear spectrum $\omega_{k} \simeq \omega_{0} \pi k / N$. We define $K$ as the approximated fraction of modes in the linear part of the spectrum, a number that scales as $K \propto N$. At low frequency, the numerator of Eq. (10) converges to $1\left(\omega_{k} \ll \min \left[\omega_{m}, \omega_{J}\right]\right)$. Then, for $\omega>0$ and provided that the imaginary part is much smaller than the lowest eigenfrequency $\varepsilon \ll \omega_{k=1}$ (which is equivalent to the requirement that $N \varepsilon=$ const or $K \varepsilon=$ const), one can approximate the real part of the admittance to a sum of Lorentzian functions

$$
\operatorname{Re}\left[Y_{\text {har }}(\omega)\right] \simeq \frac{1}{Z_{0}}\left(\frac{\pi \omega_{0}}{N}\right) \sum_{k=1}^{K} \frac{\omega}{\omega_{k}} \frac{\varepsilon / \pi}{\left(\omega_{k}-\omega\right)^{2}+\varepsilon^{2}},
$$




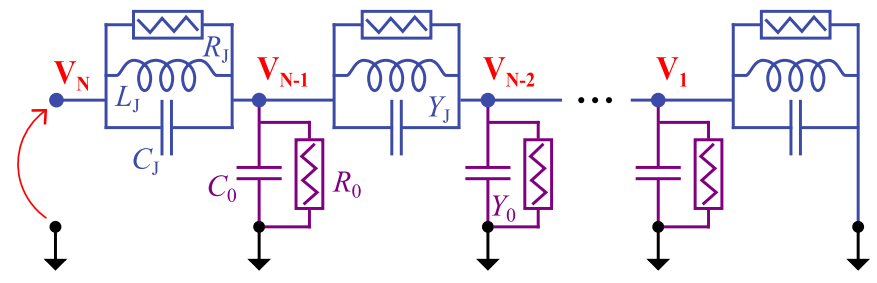

FIG. 3. Circuit model for the JJ chain with intrinsic dissipation, with shunt resistances $R_{J}$ in parallel with each junction, and $R_{0}$ in parallel with the capacitance to the ground.

with the characteristic impedance of the line

$$
Z_{0}=\sqrt{L_{J} / C_{0}} .
$$

Considering the limit of infinite length of the chain $N \rightarrow \infty$ (or equivalently $K \rightarrow \infty$ ) and keeping constant the product $N \varepsilon$ (or $K \varepsilon$ ), the admittance of the chain, given by Eq. (11), converge to an ohmic behavior $1 / Z_{0}$.

\section{B. Ohmic admittance of a finite-size dissipative $\mathbf{J J}$ chain}

In the following, we use a different approach compared to the previous section to introduce dissipation in the JJ chain. We review two types of dissipative 1D JJ chains, where the dissipation can either be intrinsically associated with all circuit elements (see Fig. 3 and Sec. IIB 1), or it can be added in a controlled manner, in parallel with the ground capacitance $C_{0}$, using a coupling inductor $L_{C}$ (see Fig. 5 and Sec. II B 2). In both cases, the chain is composed of the effective junction admittances $\mathbb{Y}_{J}(\omega)$ and the effective admittances to the ground $\mathbb{Y}_{0}(\omega)$. Applying the current conservation at each node, similarly to Eq. (1), we obtain

$$
\mathbb{Y}_{J}(\omega)\left(v_{n}-v_{n-1}\right)=\mathbb{Y}_{J}(\omega)\left(v_{n-1}-v_{n-2}\right)+\mathbb{Y}_{0}(\omega) v_{n} .
$$

Then one can repeat identically the steps following Eq. (1) in the previous section to obtain the admittance

$$
\mathbb{Y}_{\mathrm{ch}}(\omega)=\frac{\mathbb{Y}_{J}(\omega)}{N}\left(1+\sum_{k=1}^{N-1} \frac{\mathbb{Y}_{J}(\omega) \mathbb{Y}_{0}(\omega)\left[1+\cos \left(\frac{\pi k}{N}\right)\right]}{\mathbb{Y}_{0}(\omega)+2 \mathbb{Y}_{J}(\omega)\left[1-\cos \left(\frac{\pi k}{N}\right)\right]}\right)
$$

In the next two subsections, we apply this result to two hypothetic circuit implementations: (i) the case of intrinsic dissipation associated with any real superconducting circuit element, and (ii) a particular implementation of engineered dissipation, using resistive on-chip thin films. We refer to them as intrinsic dissipation and engineered dissipation, respectively.

\section{JJ chain with intrinsic dissipation}

We introduce dissipation by considering the inductances and capacitances to be nonideal elements, indicated by the resistances $R_{J}$ and $R_{0}$ in the circuit model of Fig. 3. $R_{J}$ takes into account the finite dissipation in a single JJ, potentially associated with (nonequilibrium) quasiparticles above the superconducting gap $[83,84]$ or other imperfections of the JJ dielectric barrier [85]. Similarly, $R_{0}$ accounts for dielectric losses in $C_{0}$. Then we have

$$
\begin{gathered}
\mathbb{Y}_{J}(\omega)=i \omega C_{J}+1 /\left(i \omega L_{J}\right)+1 / R_{J}, \\
\mathbb{Y}_{0}(\omega)=i \omega C_{0}+1 / R_{0}
\end{gathered}
$$

Focusing on the limit in which the two shunt resistances are much larger than the characteristic resistance of the chain, $R_{J}, R_{0} \gg Z_{0}$, following a method analogous to the one used in the previous sections, one can find the following approximate expression for the $1 \mathrm{D} \mathrm{JJ}$ chain admittance:

$$
\mathbb{Y}_{J J}^{(0)}(\omega) \simeq \frac{i 2 \omega}{N L_{J}} \mathbb{A}^{(0)}(\omega)\left(1-\frac{\omega^{2}}{\omega_{J}^{2}}\right) \sum_{k=1}^{N-1} \frac{\left(1-\frac{\omega_{k}^{2}}{\omega_{m}^{2}}\right)}{\omega_{k}^{2}-\omega^{2}-i \omega \eta_{k}^{(0)}},
$$

with the spectrum $\omega_{k}$ given by Eqs. (8) and (9). As expected, Eqs. (17) and (10) have a similar form [86]. The complex amplitude $\mathbb{A}^{(0)}(\omega)=1-i /\left(\omega R_{0} C_{0}\right)$ in Eq. (17) reduces to $\sim 1$ at frequency $\omega R_{0} C_{0} \gg 1$, and the damping coefficients for each mode $k$ are given by

$$
\eta_{k}^{(0)}=\frac{1}{R_{0} C_{0}}+\left(\frac{1}{R_{J} C_{J}}-\frac{1}{R_{0} C_{0}}\right) \frac{\omega_{k}^{2}}{\omega_{J}^{2}} .
$$

Notice that the damping is independent of the eigenmode number for frequencies $\omega_{k} \ll \omega_{J}$.

For $N \gg 1$, it is expected that the admittance of the system saturates to a smooth function of frequency. The typical length at which the discreteness of the modes disappears is reached when

$$
N \gg \pi R_{0} / Z_{0},
$$

i.e., the spacing between the low-frequency modes is much smaller than the width of the individual peaks. Since in typical JJ chains $R_{0} \sim 100 \mathrm{M} \Omega$ [17], with a characteristic impedance of the JJ chain $Z_{0} \sim \mathrm{k} \Omega$, from Eq. (19) we get a minimum required number of junctions $N \gtrsim 10^{5}$, a number that is difficult to achieve in experimental JJ devices.

In Fig. 4 we plot the calculated real part of the JJ chain admittance, following Eq. (17), for $N=10^{5}$. The inset shows the same calculation for a short chain with $N=25$ to evidence the discrete mode structure of the JJ chain admittance. For $N=10^{5}$, the admittance at low frequency still shows large-amplitude oscillations caused by the discreteness of the eigenmodes spectrum, pointing out that even longer chains are needed to achieve an ohmic behavior in JJ chains with intrinsic dissipation.

\section{JJ chain with engineered dissipation}

Hereafter, we neglect the large intrinsic resistances $R_{J}$ and $R_{0}$ associated with the dissipative part of nonideal capacitances and inductances. As shown in Fig. 5, we assume $\mathbb{Y}_{J}(\omega)$ to be a pure imaginary admittance, whereas the element $\mathbb{Y}_{0}(\omega)$ is constructed using an ideal capacitance $C_{C}$, in parallel with the series combination $R_{C}$ and $L_{C}$,

$$
\begin{gathered}
\mathbb{Y}_{J}(\omega)=i \omega C_{J}+1 /\left(i \omega L_{J}\right), \\
\mathbb{Y}_{0}(\omega)=i \omega C_{C}+\frac{1}{R_{C}+i \omega L_{C}} .
\end{gathered}
$$




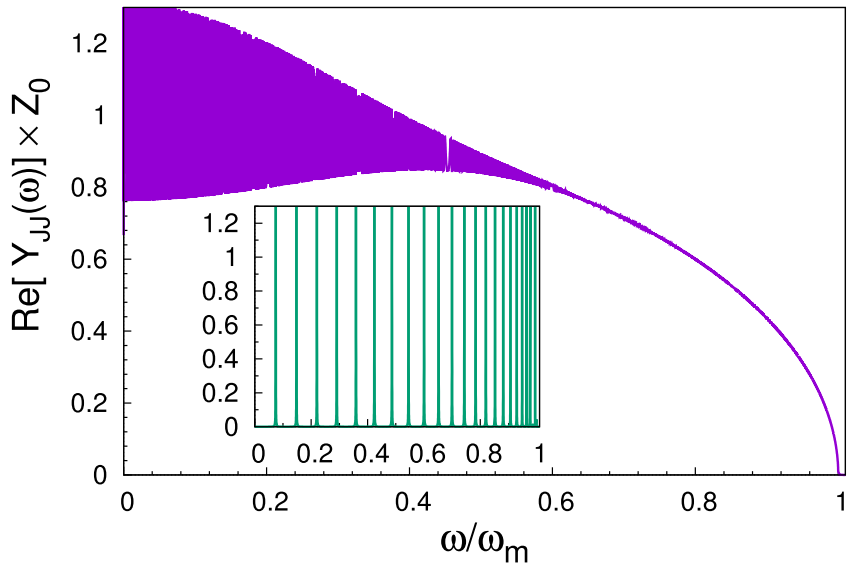

FIG. 4. The admittance of the $1 \mathrm{D} J \mathrm{~J}$ chain with intrinsic dissipation [following Eq. (17)] for $N=10^{5}$ and parameters $C_{0} / C_{J}=10$, $R_{0}=R_{J}=5 \times 10^{4} Z_{0}$. For clarity, the inset shows the results for a much shorter chain, with $N=25$.

The inductance $L_{C}$ opens a gap in the spectrum and the eigenmodes are now given by

$$
\Omega_{k}=\frac{2}{\sqrt{L_{J} C_{C}}} \sqrt{\frac{\sin ^{2}[\pi k /(2 N)]+L_{J} /\left(4 L_{C}\right)}{1+\left(4 C_{J} / C_{C}\right) \sin ^{2}[\pi k /(2 N)]}},
$$

with the maximum frequency of the spectrum given by $\omega_{m}=2 \sqrt{\left[1 / L_{J}+1 /\left(4 L_{C}\right)\right] /\left(C_{C}+4 C_{J}\right)}$, and the minimum frequency $\omega_{c}=1 / \sqrt{L_{C} C_{C}}$ for $N \gg \max \left[1, \pi \sqrt{C_{J} / C_{C}}\right]$. It is also convenient to introduce the characteristic impedance

$$
Z_{C}=\sqrt{L_{J} / C_{C}} .
$$

Focusing on the frequency range containing the spectrum, $\omega_{c}<\omega<\omega_{m}$, and in the regime

$$
C_{J} \ll C_{C}, \quad L_{J} \ll L_{C}, \quad \frac{R_{C}}{Z_{C}} \sqrt{\frac{L_{J}}{L_{C}}} \ll 1,
$$

using the method of Sec. II A, we obtain an approximate expression for the real part of the admittance of the chain:

$$
\mathbb{Y}_{\mathrm{JJ}}(\omega) \simeq \frac{i 2 \omega}{N L_{J}} \mathbb{A}(\omega)\left(1-\frac{\omega^{2}}{\omega_{J}^{2}}\right) \sum_{k=1}^{N-1} \frac{\left(1-\frac{\Omega_{k}^{2}}{\omega_{m}^{2}}\right)}{\Omega_{k}^{2}-\omega^{2}-i \omega \eta_{k}(\omega)}
$$

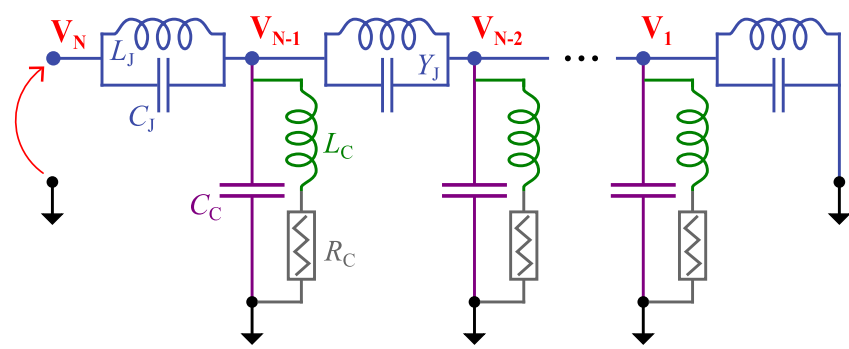

FIG. 5. Circuit model for the JJ chain with engineered dissipation. The dissipative element $R_{C}$ is introduced using a coupling inductor $L_{C}$, in parallel with the coupling capacitor $C_{C}$ connected to the ground.

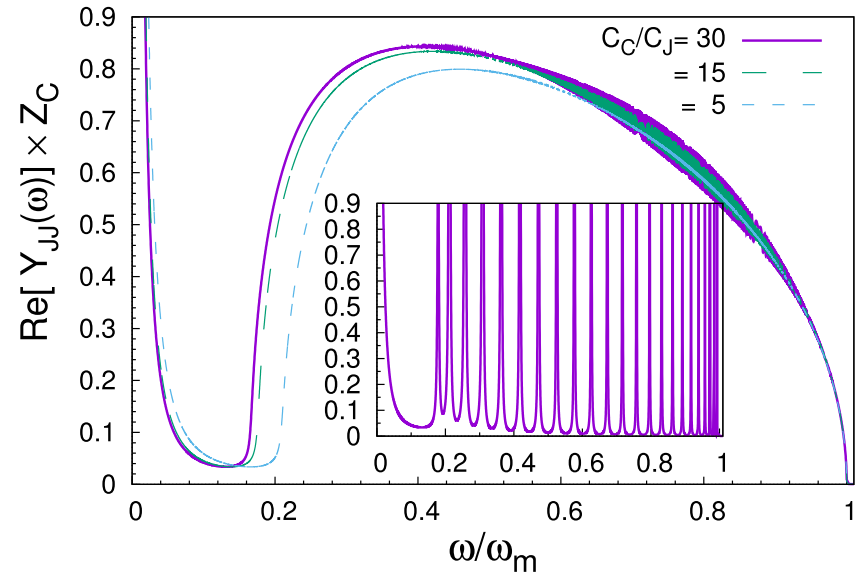

FIG. 6. The admittance of the 1D JJ chain with engineered dissipation [following Eq. (25)] for $N=5000$ and parameters $L_{C} / L_{J}=$ $10, R_{C} / Z_{C}=0.1$, and different ratios $C_{C} / C_{J}$. For clarity, the inset shows the results for a much shorter chain, with $N=25$, for $C_{C} / C_{J}=$ 30 .

The complex amplitude $\mathbb{A}(\omega)$ and the functions $\eta_{k}(\omega)$ are now given by

$$
\begin{gathered}
\mathbb{A}(\omega)=\left(1-\frac{\omega_{c}^{2}}{\omega^{2}}-\frac{i R_{C}}{\omega L_{C}}\right)\left[\frac{1+L_{J} /\left(4 L_{C}\right)}{1-C_{J} C_{C} /\left(L_{J} L_{C}\right)}\right], \\
\eta_{k}(\omega)=\frac{R_{C} / L_{C}}{1-C_{J} L_{J} /\left(C_{C} L_{C}\right)}\left(1-\frac{\Omega_{k}^{2}-\omega_{c}^{2}}{\omega^{2}}\right) .
\end{gathered}
$$

Notice that the damping coefficients for each mode are frequency-dependent, and they increase up to $R_{C} / L_{C}$ as the frequency decreases toward the minimum frequency of the spectrum. As we will show in the next paragraph, the resulting increase of the spectral linewidth of the modes at lower frequencies allows a decrease by two orders of magnitude of the minimum required $N$, compared to the previous section.

In the limit $N \gg \pi \sqrt{L_{C} / L_{J}}$, we can estimate the length above which the discreteness of the modes disappears in the admittance,

$$
N \gg \pi\left(L_{C} / L_{J}\right)^{\frac{3}{4}} \sqrt{Z_{C} / R_{C}}
$$

For experimentally feasible parameters such as $R_{C}=50 \Omega$, $Z_{C} \sim \mathrm{k} \Omega$, and $L_{C} / L_{J}=10$, the minimum required number of JJs is $N \sim 10^{3}$. Using these parameters, in Fig. 6 we plot the calculated real part of the $1 \mathrm{D} \mathrm{JJ}$ chain admittance according to Eq. (25) for $N=5000$. We observe a smooth behavior of the admittance in a wide frequency range, although some oscillations due to the granularity of the spectrum still appear in the high-frequency range. These oscillations are a consequence of the fact that the mode damping decreases as the frequency approaches the upper edge of the spectrum; see Eq. (27).

We conclude this section by emphasizing that the introduction of dissipation in a $1 \mathrm{D} \mathrm{JJ}$ chain via a coupling inductor to the ground (see Fig. 5) allows the design of quasi-ohmic dissipative environments, functioning in a relatively wide band. The required system parameters, such as chain lengths in the range of $10^{3}$, although ambitious, are not unrealistic for state-of-the-art JJ technology. 


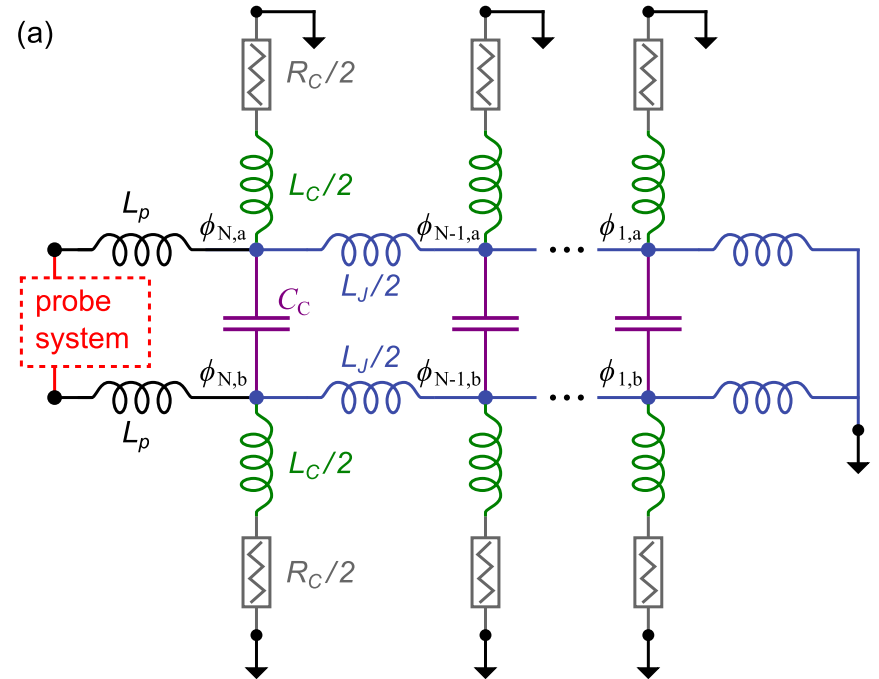

(b)

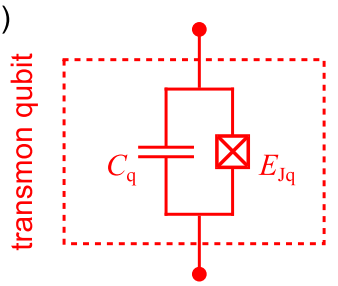

(c)

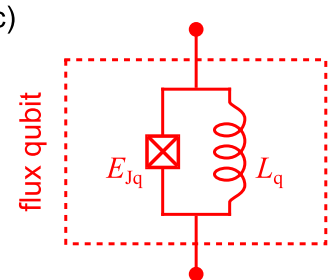

FIG. 7. (a) Equivalent circuit of the PJJC shown in Fig. 1. The microstrip transmission line is modeled as a resistance $R_{C}$. This floating configuration is convenient to connect the PJJA impedance to a probe system such as a transmon (b) or flux (c) qubit.

\section{ADMITTANCE OF THE DOUBLE CHAIN WITH ENGINEERED DISSIPATION}

Following the design of a 1D JJ chain with engineered dissipation introduced in Fig. 5, in this section we discuss a similar proposal, the PJJC device shown in Fig. 1, where dissipation is not added via on-chip dissipative elements, like in Sec. II B 2, but rather by a uniform coupling to a microwave transmission line, which could also allow the continuous monitoring of the dissipated energy.

We analyze theoretically an equivalent circuit of the PJJC, as shown in Fig. 7, which captures one essential ingredient of the PJJC proposal, namely a uniform dissipation distributed along the nodes of the chain. The resulting PJJA impedance can be connected to a probe system, for example a flux $[87,88]$ or transmon [89] qubit, coupled via the inductance $L_{P}$. The microstrip transmission line, which is ideally reflectionless and matched to a standard coaxial cable $(50 \Omega)$, acts as a resistor $R_{C}$ at each node of the chain. Under the condition of local mirror reflection symmetry for the two chains, we show that the PJJC is equivalent to a single chain connected directly to the ground via $C_{C}$, as shown in Fig. 5. Hereafter, we assume the relevant regime $C_{C} \gg C_{J}$ and neglect the junction capacitance $C_{J}$ to simplify the formulas, although the treatment can be extended to the case $C_{J} \neq 0$. Similarly, we consider the local ground capacitance of each island negligible, i.e., $C_{0} \ll C_{C}$.

We quantize the circuit of Fig. 7 using the standard method to construct the Lagrangian and equations of motion for a quantum electromagnetic circuit formed by lumped elements
[90]. We use the phase nodes variables $\Phi_{n, s}$, with $n=1, \ldots, N$ and $s=a, b$ for the two chains connected via the capacitances $C_{C}$. The index $n$ runs from $n=1, \ldots, N-1$ for the two chains, with the boundary condition $\Phi_{0}=0$. The index $n=N$ is for the probe system, the qubit, formally described by the node phases $\Phi_{N, a}, \Phi_{N, b}$. For $\Phi_{n, s}$, with $n=1, \ldots, N-1$, the dynamics of the system is ruled by the equations of motion

$$
\begin{aligned}
C_{C} \frac{d^{2}\left(\Phi_{n, a}-\Phi_{n, b}\right)}{d t^{2}}= & -\frac{2}{L_{J}}\left(2 \Phi_{n, a}-\Phi_{n-1, a}-\Phi_{n+1, a}\right) \\
& -\int_{-\infty}^{+\infty} d t^{\prime} 2 Y_{c}\left(t-t^{\prime}\right) \frac{d \Phi_{n, a}}{d t^{\prime}}
\end{aligned}
$$

and

$$
\begin{aligned}
C_{C} \frac{d^{2}\left(\Phi_{n, b}-\Phi_{n, a}\right)}{d t^{2}}= & -\frac{2}{L_{J}}\left(2 \Phi_{n, b}-\Phi_{n-1, b}-\Phi_{n+1, b}\right) \\
& -\int_{-\infty}^{+\infty} d t^{\prime} 2 Y_{c}\left(t-t^{\prime}\right) \frac{d \Phi_{n, b}}{d t^{\prime}},
\end{aligned}
$$

with the external admittance

$$
Y_{c}(t)=\theta(t) e^{-t / \tau_{c}} / L_{C}, \quad Y_{c}(\omega)=1 /\left(R_{C}+i \omega L_{C}\right),
$$

where $\theta(t)$ is the theta function, and $\tau_{c}=L_{C} / R_{C}$. It is now convenient to introduce the phase differences

$$
\phi_{n}=\Phi_{n, a}-\Phi_{n, b} .
$$

We can also define equivalently the variables corresponding to the average local phase $\sim \Phi_{n, a}+\Phi_{n, b}$. We remark that a finite $C_{J}$ does not introduce any coupling between the two families of modes. Then, taking the difference between the equations of motion (29) and (30), we get

$$
\begin{aligned}
C_{C} \frac{d^{2} \phi_{n}}{d t^{2}}= & -\frac{1}{L_{J}}\left(2 \phi_{n}-\phi_{n-1}-\phi_{n+1}\right) \\
& -\int_{-\infty}^{+\infty} d t^{\prime} Y_{c}\left(t-t^{\prime}\right) \frac{d \phi_{n}}{d t^{\prime}} .
\end{aligned}
$$

We remark that, for a system characterized by local capacitances $C_{C}^{(n)}$ and inductances $L_{J}^{(n, a)}, L_{J}^{(n, b)}$, Eq. (33) remains valid if mirror reflection symmetry is present. The set of equations (33), valid for $n=1, \ldots, N-1$, can be cast in the following matrix form:

$$
\begin{aligned}
C_{C} \frac{d^{2} \vec{\phi}^{\prime}}{d t^{2}}= & -\frac{\overline{\bar{M}}_{\mathrm{TB}}}{L_{J}} \vec{\phi}^{\prime}-\int_{-\infty}^{+\infty} d t^{\prime} Y_{c}\left(t-t^{\prime}\right) \frac{d \vec{\phi}^{\prime}\left(t^{\prime}\right)}{d t^{\prime}} \\
& +\frac{1}{L_{J}}\left[\begin{array}{c}
0 \\
0 \\
\ldots \\
\phi_{N}
\end{array}\right],
\end{aligned}
$$

with the vector ${ }^{T} \vec{\phi}^{\prime}=\left(\phi_{1}, \ldots, \phi_{n}, \ldots, \phi_{N-1}\right)$, and the tightbinding matrix $\left(\overline{\bar{M}}_{\mathrm{TB}}\right)_{n m}=2 \delta_{n m}-\delta_{n-1, m}-\delta_{n+1, m}$. After the unitary transformation $\theta_{k}=\sum_{n=1}^{N-1} e_{k}(n) \phi_{n}$ that diagonalizes the tight-binding matrix $\left(\overline{\bar{M}}_{\mathrm{TB}}\right)_{n m}$, with eigenvalues and eigenvectors

$$
\lambda_{k}=2\left[1-\cos \left(\frac{\pi k}{N}\right)\right], \quad e_{k}(n)=\sqrt{\frac{2}{N}} \sin \left(\frac{\pi k n}{N}\right)
$$


for $k=1, \ldots, N-1$, we obtain the equation

$C_{C} \frac{d^{2} \theta_{k}}{d t^{2}}=-\frac{\lambda_{k}}{L_{J}} \theta_{k}-\int_{-\infty}^{+\infty} d t^{\prime} Y_{c}\left(t-t^{\prime}\right) \frac{d \theta_{k}}{d t^{\prime}}+\frac{e_{k}(N-1)}{L_{J}} \phi_{N}$.

Notice that the dissipative term is not changed after the transformation from the local node variables to the harmonic modes of the double chain. Going in the frequency space via Fourier transform, we have an inhomogeneous solution of Eq. (36),

$$
\theta_{k}(\omega)=\chi_{k}(\omega) e_{k}(N-1) \phi_{N}(\omega)
$$

with the dimensionless susceptibility

$\chi_{k}(\omega)=\frac{\left(\frac{i}{\tau_{c}}-\omega\right) /\left(L_{J} C_{C}\right)}{\omega^{3}-\frac{i}{\tau_{c}} \omega^{2}-\Omega_{k}^{2} \omega+\frac{i}{\tau_{c}}\left(\Omega_{k}^{2}-\omega_{c}^{2}\right)} \equiv \sum_{i=1}^{3} \frac{A_{i}^{(k)}}{\omega-z_{i}^{(k)}}$,

where the eigenfrequencies of the modes $\Omega_{k}$ are given by Eq. (22), $z_{i}^{(k)}$ correspond to the roots of the cubic in the denominator of $\chi_{k}(\omega)$ in Eq. (38), and the factors $A_{i}^{(k)}$ are given in Appendix A. After some algebra (see Appendix A for details), we can express the solution

$$
\begin{aligned}
\theta_{k}(t)= & -e_{k}(N-1) \int_{-\infty}^{+\infty} d t^{\prime} \theta\left(t-t^{\prime}\right) \sum_{i=1}^{3} \frac{A_{i}^{(k)}}{z_{i}^{k}} e^{i z_{i}^{(k)}\left(t-t^{\prime}\right)} \frac{d \phi_{N}}{d t^{\prime}} \\
& +\frac{e_{k}(N-1)}{\lambda_{k}} \phi_{N}(t) .
\end{aligned}
$$

Finally, we consider the equation for the node associated with the probe system (the qubit, at node $n=N$ ) in terms of the phase difference $\phi_{N}$. For simplicity, we set $L_{P}=L_{J}$ and write

$$
\begin{aligned}
\frac{d}{d t}\left(\frac{\partial \mathcal{L}_{q}}{\partial \dot{\phi}_{N}}\right) & =\frac{\partial \mathcal{L}_{q}}{\partial \phi_{N}}-\frac{1}{L_{J}}\left(\phi_{N}-\phi_{N-1}\right) \\
& =\frac{\partial \mathcal{L}_{q}}{\partial \phi_{N}}-\frac{\phi_{N}}{L_{J}}+\frac{1}{L_{J}} \sum_{k=1}^{N-1} e_{k}(N-1) \theta_{k},
\end{aligned}
$$

with $\mathcal{L}_{q}$ the Lagrangian function of the phase difference of the qubit probe: its explicit form is not relevant for our analysis. Inserting the solution Eq. (39) into Eq. (40), we get the equation for the phase difference $\phi_{N}$ of the qubit,

$$
\frac{d}{d t}\left(\frac{\partial \mathcal{L}_{q}}{\partial \dot{\phi}_{N}}\right)=\frac{\partial \mathcal{L}_{q}}{\partial \phi_{N}}-\frac{\phi_{N}}{N L_{J}}-\int_{-\infty}^{+\infty} d t^{\prime} Y_{c h}\left(t-t^{\prime}\right) \frac{d \phi_{N}}{d t^{\prime}},
$$

in which we used the property $\sum_{k=1}^{N-1} e_{k}^{2}(N-1) / \lambda_{k}=1-$ $1 / N$, and we set the admittance of the double chain to

$$
Y_{J J}(t)=\frac{\theta(t)}{L_{J}} \sum_{k=1}^{N-1} e_{k}^{2}(N-1) \sum_{i=1}^{3} \frac{A_{i}^{(k)}}{z_{i}^{k}} e^{i z_{i}^{(k)} t} .
$$

By using some algebraic relations of the $\operatorname{root} z_{i}^{(k)}$ (see Appendix A for details), we derive the final expression for the admittance in Eq. (42) in frequency space,

$$
Y_{\mathrm{JJ}}(\omega)=\frac{i 2 \omega}{N L_{J}}\left(1-\frac{\omega_{c}^{2}}{\omega^{2}}-\frac{i}{\omega \tau_{c}}\right) \sum_{k=1}^{N-1} \frac{\left(1+\frac{L_{J}}{4 L_{C}}\right)\left(1-\frac{\Omega_{k}^{2}}{\omega_{m}^{2}}\right)}{\Omega_{k}^{2}-\omega^{2}-i \omega \gamma_{k}(\omega)},
$$

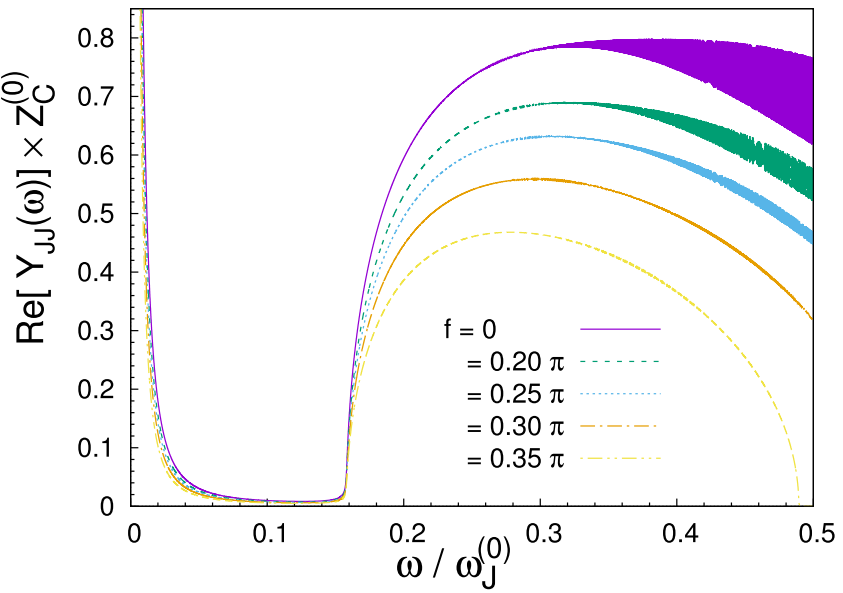

FIG. 8. The frequency-dependent admittance of the PJJC can be tuned in situ by a perpendicular magnetic field $\Phi_{B}$, threading the SQUID junctions of the PJJC device in Fig. 1. The reduced flux bias is defined as $f=2 \pi \Phi_{B} / \Phi_{0}$. The admittance and the frequency are, respectively, scaled with the characteristic impedance $Z_{C}^{(0)}=Z_{C}(0)$ and the plasma frequency $\omega_{J}^{(0)}=\omega_{J}(0)$ at zero flux $f=0$. The PJJC parameters are the following: $N=8000, C_{J} / C_{C}=0.25$, and at $f=0$ the inductance ratio $L_{C} / L_{J}^{(0)}=10$ and the resistance ratio $R_{C} / Z_{C}^{(0)}=0.025$.

with the damping functions

$$
\gamma_{k}(\omega)=\frac{1}{\tau_{c}}\left(1-\frac{\Omega_{k}^{2}-\omega_{c}^{2}}{\omega^{2}}\right) .
$$

Equations (43) and (44) represent the goal of this section: the admittance $Y_{\mathrm{JJ}}(\omega)$ corresponds exactly to the limit $C_{J} / C_{C} \rightarrow$ 0 of the admittance $\mathbb{Y}_{\mathrm{JJ}}(\omega)$ in Eq. (25) of a single chain with engineered interaction, for $L_{J} \ll L_{C}$.

To summarize, we showed that the effective circuit shown in Fig. 7 (case $C_{J} \ll C_{C}$ ) for the PJJC device of Fig. 1 is equivalent to the admittance of the single $\mathrm{JJ}$ chain with engineered dissipation discussed in Sec. IIB 2 for vanishing junction capacitance $C_{J}=0$. Therefore, in the following we will use Eqs. (25), (26), and (27) to calculate the PJCC admittance for circuits with experimentally feasible parameters.

\section{PJJC ADMITTANCE WITH EXPERIMENTALLY FEASIBLE PARAMETERS}

In the PJJC, each Josephson inductance is tuned by the applied magnetic flux as $L_{J}=L_{J}^{(0)} / \cos (f)$ with $f=2 \pi \Phi_{B} / \Phi_{0}$ the reduced magnetic flux and $L_{J}^{(0)}=\Phi_{0}^{2} /\left(8 \pi^{2} E_{J}\right)$ the zerofield inductance. The plasma frequency of the Josephson junctions, as well as the eigenmodes of the chain, are also fluxtunable. From Eq. (23) it directly follows that the characteristic impedance $Z_{C}(f)$ can be tuned in situ by the biasing field $\Phi_{B}$ through the SQUID loops composing the PJJC (see Fig. 1). An example of the scaled admittance of the PJJC for different flux biases is reported in Fig. 8. As expected, increasing the Josephson inductance by applying an external magnetic flux leads to a decrease of the admittance of the system, accompanied by a slight change in the overall frequency dependence. The frequency range in which the PJJC admittance can be considered ohmic reduces with applied flux. Depending on the 


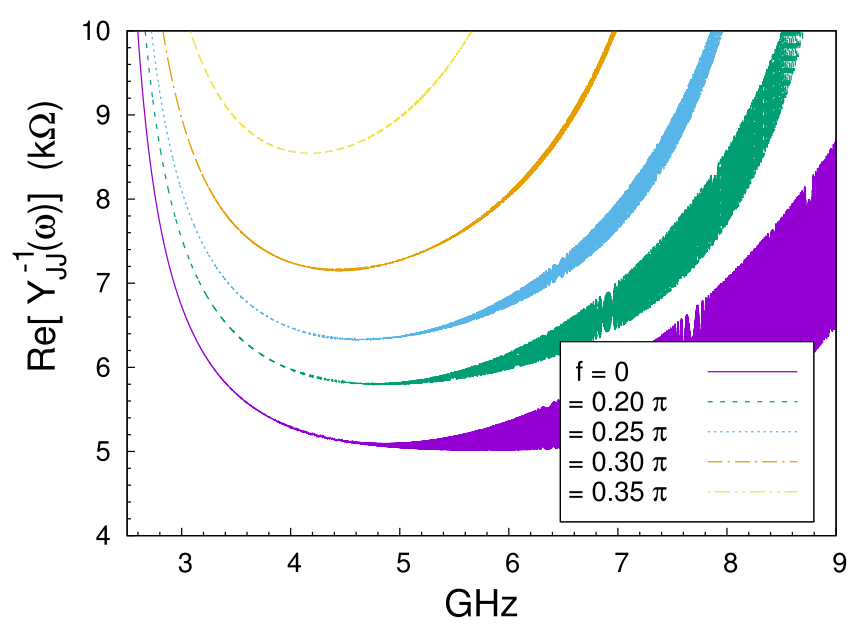

FIG. 9. The effective resistance of the PJJC electromagnetic environment shown in Fig. 1, vs frequency, for experimentally relevant circuit parameters. We chose $R_{C}=50 \Omega$ and, at flux bias $f=0$, the characteristic impedance $Z_{C}^{(0)}=4 \mathrm{k} \Omega$, with a plasma frequency $\omega_{J}^{(0)}=15 \mathrm{GHz}$. The other PJJC parameters are $N=8000$, $C_{C} / C_{J}=4$, and the inductance ratio $L_{C} / L_{J}^{(0)}=10$ at $f=0$. Notice that as we increase the flux bias $f$, the effective resistance of the environment increases to values above the resistance quantum. The value of $f$ can be increased beyond the $0.35 \pi$ threshold shown in the figure, which will further increase the effective resistance of the PJJC device. However, the frequency range where the resistance can be considered ohmic will continue to decrease, while the chain will become increasingly nonlinear.

desired application of the PJJC, one can trade by design its flux tunability for a wide bandwidth with ohmic behavior, or vice versa.

In Fig. 9 we show an example of the PJJC impedance for a circuit with experimentally feasible parameters, with a characteristic impedance $Z_{C}^{(0)}=4 \mathrm{k} \Omega$ and the plasma frequency $\omega_{J}^{(0)}=15 \mathrm{GHz}$ at $f=0$. The Josephson inductance at zero flux is $L_{J}=86 \mathrm{nH}$, the junction capacitance $C_{J}=1.3 \mathrm{fF}$, and the coupling capacitance $C_{C}=5.2 \mathrm{fF}$. The required coupling inductance $L_{C}=10 L_{J}$ is in the superinductance regime [16]. It can be implemented either using an array of JJs $[17,18]$ or a high kinetic inductance thin film, such as granular aluminum, or niobium and titanium nitrides [91-94]. Below $5 \mathrm{GHz}$, the curves appear flat in a range of $\sim 1 \mathrm{GHz}$ at $f=0$, and $\sim 0.5 \mathrm{GHz}$ for flux bias $f=0.35 \pi$. In this frequency range, the impedance of the PJJC can be tuned by the biasing magnetic field from $\sim 5 \mathrm{k} \Omega$ up to $\sim 9 \mathrm{k} \Omega$.

The single photon nonlinearity introduced by the JJs can be estimated based on Ref. [78] to be in the range of $100 \mathrm{kHz}$. This value is orders of magnitude lower than the linewidth of the PJJC modes (see the inset of Fig. 6), and it can be ignored for low power applications.

\section{SUMMARY}

We have demonstrated that the parallel Josephson junction chain device shown in Fig. 1 can implement a tunable ohmic environment over a frequency band of the order of $\mathrm{GHz}$, with an effective resistance that can be tuned through the resistance quantum $R_{q}=6.5 \mathrm{k} \Omega$. The PJJC can be connected to any two- terminal device under test, such as a superconducting qubit or a resonator, and its dissipation can be continuously monitored using a low-noise rf amplification chain.

The PJJC principle of operation can also be applied for constituent SQUIDs with different geometries, such as the ones proposed in Refs. [18,22], implementing even higher impedances and resulting in larger effective resistances. It is also worth mentioning that the rapid increase of the PJJC resistance at low frequencies (see Fig. 9) protects the device from low-energy thermal excitations.

We believe that the tunable, high-impedance ohmic environment implemented by the PJJC will be a useful instrument in the route toward quantum simulations of dissipative phase transitions, or the engineering of environments for autonomous quantum error correction schemes.

\section{ACKNOWLEDGMENTS}

We acknowledge Denis Basko for interesting discussions and useful comments. G.R. acknowledges support from by the German Excellence Initiative through the Zukunftskolleg of the University of Konstanz, the DFG through the SFB 767 and Grant No. 13971016, and the MWK-RiSC program, Project No. 13551400. I.M.P. acknowledges support from the Alexander von Humboldt Foundation in the framework of a Sofja Kovalevskaja award endowed by the German Federal Ministry of Education and Research.

\section{APPENDIX: SUSCEPTIBILITY FOR THE $\boldsymbol{k}$ HARMONIC MODES}

In this appendix, we list the main steps of the calculations leading to the main results Eqs. (43) and (44), in Sec. III, for the admittance of the double shown in Fig. 7. The solution for the dynamics of the harmonic modes connected to the probe qubit in frequency space is given by Eq. (37) with the susceptibility for the single eigenmode $k$ defined by Eq. (38) and with factors given by

$$
A_{1}^{(k)}=-\frac{\left(z_{1}^{(k)}-\frac{i}{\tau_{c}}\right) /\left(L_{J} C_{C}\right)}{\left(z_{1}^{(k)}-z_{2}^{(k)}\right)\left(z_{1}^{(k)}-z_{3}^{(k)}\right)},
$$

and similar definitions of $A_{2}^{(k)}, A_{3}^{(k)}$. The roots of the cubic satisfy Veta's relations

$$
\begin{gathered}
z_{1}^{(k)}+z_{2}^{(k)}+z_{3}^{(k)}=i / \tau_{c}, \\
z_{1}^{(k)} z_{2}^{(k)}+z_{2}^{(k)} z_{3}^{(k)}+z_{1}^{(k)} z_{3}^{(k)}=-\Omega_{k}^{2}, \\
z_{1}^{(k)} z_{2}^{(k)} z_{3}^{(k)}=\left(i / \tau_{c}\right)\left(\omega_{c}^{2}-\Omega_{k}^{2}\right) .
\end{gathered}
$$

We also have the sum rules

$$
\begin{gathered}
\sum_{i=1}^{3} \frac{A_{i}^{(k)}}{z_{i}^{(k)}}=\frac{1}{\omega_{c}^{2}-\Omega_{k}^{2}}, \\
\sum_{i=1}^{3} \frac{1+\frac{\omega^{2}-i \omega / \tau_{c}-\Omega_{k}^{2}}{\Omega_{k}^{2}-\omega_{c}^{2}}}{z_{i}^{(k)}\left(\omega-z_{i}^{(k)}\right)}=\frac{1 \omega_{k}^{3}-\frac{i}{\tau_{c}} \omega^{2}-\omega_{k}^{2} \omega+\frac{i}{\tau_{c}}\left(\omega_{k}^{2}-\omega_{c}^{2}\right)}{\omega^{3}} .
\end{gathered}
$$


We consider the solution in time, which reads

$$
\begin{aligned}
\theta_{k}(t) & =\alpha_{k} \sum_{i=1}^{3} i A_{i}^{(k)} \int_{-\infty}^{t} d t^{\prime} e^{i z_{i}^{(k)}\left(t-t^{\prime}\right)} \phi_{N}\left(t^{\prime}\right) \\
& =\alpha_{k} \sum_{i=1}^{3} \frac{A_{i}^{(k)}}{z_{i}^{(k)}}\left[-\phi_{N}(t)+\int_{-\infty}^{t} d t^{\prime} e^{i z_{i}^{(k)}\left(t-t^{\prime}\right)} \frac{d \phi_{N}\left(t^{\prime}\right)}{d t^{\prime}}\right]
\end{aligned}
$$

in which we set $\alpha_{k}=e_{k}(N-1)=\sqrt{2 / N} \sin [\pi k(N-1) / N]$ and we have used the fact that $\lim _{\Delta t \rightarrow-\infty} e^{i z_{i}^{(k)} \Delta t} \phi_{N}(t+\Delta t)=$ 0 since the roots have positive imaginary parts. The weighted sum over modes reduces to

$$
\sum_{k=1}^{N-1} \alpha_{k} \theta_{k}(t)=-\left(\sum_{k=1}^{N-1} \alpha_{k}^{2} \sum_{i=1}^{3} \frac{A_{i}^{(k)}}{z_{i}^{(k)}}\right) \phi_{N}(t)
$$

[1] A. A. Clerk, S. M. Girvin, F. Marquardt, and R. J. Schoelkopf, Rev. Mod. Phys. 82, 1155 (2010).

[2] M. Devorert and R. J. Schoelkopf, Science 339, 1169 (2013).

[3] U. Vool and M. Devoret, Int. J. Circ. Theor. Appl. 45, 897 (2017).

[4] B. Kraus, H. P. Büchler, S. Diehl, A. Kantian, A. Micheli, and P. Zoller, Phys. Rev. A 78, 042307 (2008).

[5] S. Diehl, A. Micheli, A. Kantian, B. Kraus, H. P. Büchler, and P. Zoller, Nat. Phys. 4, 878 (2008).

[6] F. Verstraete, M. M. Wolf, and J. Ignacio Cirac, Nat. Phys. 5, 633 (2009).

[7] H. Weimer, M. Müller, I. Lesanovsky, P. Zoller, and H. P. Büchler, Nat. Phys. 6, 382 (2010).

[8] C. C. Gerry and E. E. Hach, Phys. Lett. A 174, 185 (1993).

[9] B. M. Garraway and P. L. Knight, Phys. Rev. A 49, 1266 (1994).

[10] R. L. de Matos Filho and W. Vogel, Phys. Rev. Lett. 76, 608 (1996).

[11] J. F. Poyatos, J. I. Cirac, and P. Zoller, Phys. Rev. Lett. 77, 4728 (1996).

[12] L. D. Tóth, N. R. Bernier, A. Nunnenkamp, A. K. Feofanov, and T. J. Kippenberg, Nat. Phys. 13, 787 (2017).

[13] Z. Leghtas, U. Vool, S. Shankar, M. Hatridge, S. M. Girvin, M. H. Devoret, and M. Mirrahimi, Phys. Rev. A 88, 023849 (2013).

[14] Z. Leghtas, S. Touzard, I. M. Pop, A. Kou, B. Vlastakis, A. Petrenko, K. M. Sliwa, A. Narla, S. Shankar, M. J. Hatridge, M. Reagor, L. Frunzio, R. J. Schoelkopf, and M. Mirrahimi, Science 347, 853 (2015).

[15] C. Wang, Y. Y. Gao, P. Reinhold, R. W. Heeres, N. Ofek, K. Chou, C. Axline, M. Reagor, J. Blumoff, K. M. Sliwa, L. Frunzio, S. M. Girvin, L. Jiang, M. Mirrahimi, M. H. Devoret, and R. J. Schoelkopf, Science 352, 1087 (2016).

[16] V. E. Manucharyan, J. Koch, and L. I. Glazman, Science 326, 113 (2009).

[17] N. A. Masluk, I. M. Pop, A. Kamal, Z. K. Minev, and M. H. Devoret, Phys. Rev. Lett. 109, 137002 (2012).

[18] M. T. Bell, I. A. Sadovskyy, L. B. Ioffe, A. Y. Kitaev, and M. E. Gershenson, Phys. Rev. Lett. 109, 137003 (2012).

$$
\begin{aligned}
& +\sum_{k=1}^{N-1} \alpha_{k}^{2} \sum_{i=1}^{3} \frac{A_{i}^{(k)}}{z_{i}^{(k)}} \int_{-\infty}^{t} d t^{\prime} e^{i z_{i}^{(k)}\left(t-t^{\prime}\right)} \dot{\phi}_{N}\left(t^{\prime}\right) \\
= & \frac{N-1}{N} \phi_{N}(t)+L_{J} \int_{-\infty}^{+\infty} d t^{\prime} Y_{\mathrm{JJ}}\left(t-t^{\prime}\right) \frac{d \phi_{N}\left(t^{\prime}\right)}{d t^{\prime}},
\end{aligned}
$$

with the admittance of the chain given by

$$
Y_{\mathrm{JJ}}(t)=\frac{\theta(t)}{L_{J}} \sum_{k=1}^{N-1} \alpha_{k}^{2} \sum_{i=1}^{3} \frac{A_{i}^{(k)}}{z_{i}^{k}} e^{i z_{i}^{(k)}\left(t-t^{\prime}\right)} .
$$

In the frequency domain, the admittance reads

$$
Y_{\mathrm{JJ}}(\omega)=-\frac{i}{L_{J}} \sum_{k=1}^{N-1} \alpha_{k}^{2} \sum_{i=1}^{3} \frac{A_{i}^{(k)}}{z_{i}^{(k)}\left(\omega-z_{i}^{(k)}\right)} .
$$

Using Veta's relations Eqs. (A2)-(A4) and the sum rules Eqs. (A5) and (A6), we can obtain the main results Eqs. (43) and (44).

[19] I. M. Pop, K. Geerlings, G. Catelani, R. J. Schoelkopf, L. I. Glazman, and M. H. Devoret, Nature (London) 508, 369 (2014).

[20] L. Grünhaupt, N. Maleeva, S. T. Skacel, M. Calvo, F. LevyBertrand, A. V. Ustinov, H. Rotzinger, A. Monfardini, G. Catelani, and I. M. Pop, arXiv:1802.01858.

[21] A. Stockklauser, P. Scarlino, J. V. Koski, S. Gasparinetti, C. K. Andersen, C. Reichl, W. Wegscheider, T. Ihn, K. Ensslin, and A. Wallraff, Phys. Rev. X 7, 011030 (2017).

[22] W. Zhang, W. Huang, M. E. Gershenson, and M. T. Bell, Phys. Rev. Appl. 8, 051001 (2017).

[23] A. J. Leggett, S. Chakravarty, A. T. Dorsey, M. P. A. Fisher, A. Garg, and W. Zwerger, Rev. Mod. Phys. 59, 1 (1987).

[24] U. Weiss, Quantum Dissipative Systems, 4th ed. (World Scientific, Singapore, 2012).

[25] J. S. Penttilä, Ü. Parts, P. J. Hakonen, M. A. Paalanen, and E. B. Sonin, Phys. Rev. Lett. 82, 1004 (1999).

[26] J. S. Penttilä, P. J. Hakonen, M. A. Paalanen, Ü. Parts, and E. B. Sonin, Physica B 284, 1832 (2000).

[27] M. H. Devoret, S. Girvin, and R. Schoelkopf, Ann. Phys. 16, 767 (2007).

[28] T. Niemczyk, F. Deppe, H. Huebl, E. P. Menzel, F. Hocke, M. J. Schwarz, J. J. García-Ripoll, D. Zueco, T. Hümmer, E. Solano, A. Marx, and R. Gross, Nat. Phys. 6, 772 (2010).

[29] F. Yoshihara, T. Fuse, S. Ashhab, K. Kakuyanagi, S. Saito, and K. Semba, Nat. Phys. 13, 44 (2016).

[30] P. Forn-Díaz, J. J. García-Ripoll, B. Peropadre, J. L. Orgiazzi, M. A. Yurtalan, R. Belyansky, C. M. Wilson, and A. Lupascu Nat. Phys. 13, 39 (2017).

[31] B. Peropadre, D. Zueco, D. Porras, and J. J. García-Ripoll, Phys. Rev. Lett. 111, 243602 (2013).

[32] J. Braumüller, M. Marthaler, A. Schneider, A. Stehli, H Rotzinger, M. Weides, and A. V. Ustinov, Nat. Commun. 8, 779 (2017).

[33] J. Leppäkangas, J. Braumüller, M. Hauck, J.-M. Reiner, I. Schwenk, S. Zanker, L. Fritz, A. V. Ustinov, M. Weides, and M. Marthaler, Phys. Rev. A 97, 052321 (2018). 
[34] L. Magazzù, P. Forn-Díaz, R. Belyansky, J.-L. Orgiazzi, M. A. Yurtalan, M. R. Otto, A. Lupascu, C. M. Wilson, and M. Grifoni, Nat. Commun. 9, 1403 (2018).

[35] J. P. Martinez, S. Leger, N. Gheereart, R. Dassonneville, L. Planat, F. Foroughi, Y. Krupko, O. Buisson, C. Naud, W. Guichard, S. Florens, I. Snyman, and N. Roch, arXiv: 1802.00633.

[36] M. Goldstein, M. H. Devoret, M. Houzet, and L. I. Glazman, Phys. Rev. Lett. 110, 017002 (2013).

[37] R. Yagi, S.-i. Kobayashi, and Y. Ootuka, J. Phys. Soc. Jpn. 66, 3722 (1997).

[38] C. H. van der Wal, F. K. Wilhelm, C. J. P. M. Harmans, and J. E. Mooij, Europhys. J. B 31, 111 (2003).

[39] P. J. Jones, J. A. M. Huhtamäki, J. Salmilehto, K. Y. Tan, and M. Möttönen, Sci. Rep. 3, 1987 (2013).

[40] F. Pastawski, L. Clemente, and J. I. Cirac, Phys. Rev. A 83, 012304 (2011).

[41] J. Tuorila, M. Partanen, T. Ala-Nissila, and M. Möttönen, npj Quantum Inf. 3, 27 (2017).

[42] M. Mirrahimi, Z. Leghtas, V. V. Albert, S. Touzard, R. J. Schoelkopf, L. Jiang, and M. H. Devoret, New J. Phys. 16, 045014 (2014).

[43] J. Cohen and M. Mirrahimi, Phys. Rev. A 90, 062344 (2014).

[44] J. Cohen, W. C. Smith, M. H. Devoret, and M. Mirrahimi, Phys. Rev. Lett. 119, 060503 (2017).

[45] J. Clarke and F. K. Wilhelm, Nature (London) 453, 1031 (2008).

[46] J. M. Martinis, Quantum Inf. Proc. 8, 81 (2009).

[47] G. Wendin, Rep. Prog. Phys. 80, 106001 (2017).

[48] Z.-L. Xiang, S. Ashhab, J. You, and F. Nori, Rev. Mod. Phys. 85, 623 (2013).

[49] J. M. Pirkkalainen, S. U. Cho, J. Li, G. S. Paraoanu, P. J. Hakonen, and M. A. Sillanpää, Nature (London) 494, 211 (2013).

[50] M. Hofheinz, F. Portier, Q. Baudouin, P. Joyez, D. Vion, P. Bertet, P. Roche, and D. Esteve, Phys. Rev. Lett. 106, 217005 (2011).

[51] M. C. Cassidy, A. Bruno, S. Rubbert, M. Irfan, J. Kammhuber, R. N. Schouten, A. R. Akhmerov, and L. P. Kouwenhoven, Science 355, 939 (2017).

[52] R. Fazio, Phys. Rep. 355, 235 (2001).

[53] H. S. J. van der Zant, C. J. Muller, L. J. Geerligs, C. J. P. M. Harmans, and J. E. Mooij, Phys. Rev. B 38, 5154 (1988).

[54] K. A. Matveev, A. I. Larkin, and L. I. Glazman, Phys. Rev. Lett. 89, 096802 (2002).

[55] I. M. Pop, I. Protopopov, F. Lecocq, Z. Peng, B. Pannetier, O. Buisson, and W. Guichard, Nat. Phys. 6, 589 (2010).

[56] F. Maibaum, S. V. Lotkhov, and A. B. Zorin, Phys. Rev. B 84, 174514 (2011).

[57] I. M. Pop, B. Douçot, L. Ioffe, I. Protopopov, F. Lecocq, I. Matei, O. Buisson, and W. Guichard, Phys. Rev. B 85, 094503 (2012).

[58] V. E. Manucharyan, N. A. Masluk, A. Kamal, J. Koch, L. I. Glazman, and M. H. Devoret, Phys. Rev. B 85, 024521 (2012).

[59] G. Rastelli, I. M. Pop, and F. W. J. Hekking, Phys. Rev. B 87, 174513 (2013).

[60] R. Süsstrunk, I. Garate, and L. I. Glazman, Phys. Rev. B 88, 060506 (2013).

[61] A. Ergül, J. Lidmar, J. Johansson, Y. Azizoğlu, D. Schaeffer, and D. B. Haviland, New J. Phys. 15, 095014 (2013).
[62] D. A. Garanin and E. M. Chudnovsky, Phys. Rev. B 93, 094506 (2016).

[63] A. Ergül, T. Weiß1, J. Johansson, J. Lidmar, and D. B. Haviland, Sci. Rep. 7, 11447 (2017).

[64] Z. Hermon, E. Ben-Jacob, and G. Schön, Phys. Rev. B 54, 1234 (1996).

[65] D. B. Haviland and P. Delsing, Phys. Rev. B 54, R6857 (1996).

[66] S. Corlevi, W. Guichard, F. W. J. Hekking, and D. B. Haviland, Phys. Rev. B 74, 224505 (2006).

[67] S. V. Syzranov, K. B. Efetov, and B. L. Altshuler, Phys. Rev. Lett. 103, 127001 (2009).

[68] J. Homfeld, I. Protopopov, S. Rachel, and A. Shnirman, Phys. Rev. B 83, 064517 (2011).

[69] N. Vogt, R. Schäfer, H. Rotzinger, W. Cui, A. Fiebig, A. Shnirman, and A. V. Ustinov, Phys. Rev. B 92, 045435 (2015).

[70] K. Cedergren, S. Kafanov, J. L. Smirr, J. H. Cole, and T. Duty, Phys. Rev. B 92, 104513 (2015).

[71] K. Cedergren, R. Ackroyd, S. Kafanov, N. Vogt, A. Shnirman, and T. Duty, Phys. Rev. Lett. 119, 167701 (2017).

[72] A. L. Rakhmanov, A. M. Zagoskin, S. Savel'ev, and F. Nori, Phys. Rev. B 77, 144507 (2008).

[73] C. Hutter, E. A. Tholén, K. Stannigel, J. Lidmar, and D. B. Haviland, Phys. Rev. B 83, 014511 (2011).

[74] D. Zueco, J. J. Mazo, E. Solano, and J. J. García-Ripoll, Phys. Rev. B 86, 024503 (2012).

[75] B. Peropadre, D. Zueco, F. Wulschner, F. Deppe, A. Marx, R. Gross, and J. J. García-Ripoll, Phys. Rev. B 87, 134504 (2013).

[76] M. Taguchi, D. M. Basko, and F. W. J. Hekking, Phys. Rev. B 92, 024507 (2015).

[77] M. A. Iontsev, S. I. Mukhin, and M. V. Fistul, Phys. Rev. B 94, 174510 (2016).

[78] T. Weiß1, B. Küng, E. Dumur, A. K. Feofanov, I. Matei, C. Naud, O. Buisson, F. W. J. Hekking, and W. Guichard, Phys. Rev. B 92, 104508 (2015).

[79] P. R. Muppalla, O. Gargiulo, S. I. Mirzaei, B. Prasanna Venkatesh, M. L. Juan, L. Grünhaupt, I. M. Pop, and G. Kirchmair, Phys. Rev. B 97, 024518 (2018).

[80] C. Eichler, Y. Salathé, J. Mlynek, S. Schmidt, and A. Wallraff, Phys. Rev. Lett. 113, 110502 (2014).

[81] G. Rastelli, M. Vanević, and W. Belzig, New J. Phys. 17, 053026 (2015).

[82] In this work, the boundary condition is that the chain is connected to the ground at the end, so that the wave functions of the modes vanish at this point. This explains the difference of a factor 2 inside the cos function for the eigenfrequencies of the chain studied in this work with respect to the frequency of the harmonic modes in a Josephson junction ring studied in Refs. [81] and [59].

[83] U. Vool, I. M. Pop, K. Sliwa, B. Abdo, C. Wang, T. Brecht, Y. Y. Gao, S. Shankar, M. Hatridge, G. Catelani, M. Mirrahimi, L. Frunzio, R. J. Schoelkopf, L. I. Glazman, and M. H. Devoret, Phys. Rev. Lett. 113, 247001 (2014).

[84] C. Wang, Y. Y. Gao, I. M. Pop, U. Vool, C. Axline, T. Brecht, R. W. Heeres, L. Frunzio, G. Catelani, L. I. Glazman, and R. J. Schoelkopf, Nat. Commun. 5, 5836 (2014).

[85] C. Wang, C. Axline, Y. Y. Gao, T. Brecht, Y. Chu, L. Frunzio, M. H. Devoret, and R. J. Schoelkopf, Appl. Phys. Lett. 107, 162601 (2015) 
[86] Equation (17) still contains a part of renormalized, highfrequency capacitance, in contrast to Eq. (10), which includes only the contribution of the harmonic modes. Since we are interested in the real part in $\operatorname{Re}\left[\mathbb{Y}_{\mathrm{JJ}}(\omega)\right]$, this difference is not important.

[87] J. E. Mooij, Science 285, 1036 (1999).

[88] I. Chiorescu, Science 299, 1869 (2003).

[89] J. A. Schreier, A. A. Houck, J. Koch, D. I. Schuster, B. R. Johnson, J. M. Chow, J. M. Gambetta, J. Majer, L. Frunzio, M. H. Devoret, S. M. Girvin, and R. J. Schoelkopf, Phys. Rev. B 77, 180502 (2008).

[90] M. H. Devoret, in Quantum Fluctuations (Les Houches Session LXIII) (Elsevier, Amsterdam, 2002), p. 1.
[91] H. Rotzinger, S. T. Skacel, M. Pfirrmann, J. N. Voss, J. Münzberg, S. Probst, P. Bushev, M. P. Weides, A. V. Ustinov, and J. E. Mooij, Supercond. Sci. Technol. 30, 025002 (2017).

[92] A. J. Annunziata, D. F. Santavicca, L. Frunzio, G. Catelani, M. J. Rooks, A. Frydman, and D. E. Prober, Nanotechnology 21, 445202 (2010).

[93] N. Samkharadze, A. Bruno, P. Scarlino, G. Zheng, D. P. DiVincenzo, L. DiCarlo, and L. M. K. Vandersypen, Phys. Rev. Appl. 5, 044004 (2016).

[94] M. R. Vissers, J. Gao, D. S. Wisbey, D. A. Hite, C. C. Tsuei, A. D. Córcoles, M. Steffen, and D. P. Pappas, Appl. Phys. Lett. 97, 232509 (2010). 